\title{
Síndrome de Morquio
}

Morquio, pedíatra uruguayo, en trabajos pubircados en 1929 (1) y en 1935 (2), describio cl sindrome que lleva su nombre. En una fanilia estudió a 4 hermanos afectados, sus padres eran normales y primos en primer grado; Io califjco como una distrofia ósea familiar.

En el mismo año, 1929, Braisford, (5) rađílogo inglés, describió, originalmente, un paciente de 4 años de edad, con el nombre de osteocondrodistrofia. Actualmente, sobre las bases de estudjos clínicos, radiológicos y bioquímicos, se ha establecido que el Síndrome de Morquio es una osteocondrodistrofia hereditaria, que provoca lesiones epifisiales generalizadas. Se ha reconocido como un trastorno del tejido conectivo, implicando con esto un error congénito del metabolismo de los mucopolisacáridos, en particular del keratosulfato. Aclarando esta definición, osteocondrodistrofia, $(1,2,3,4,5,6)$ es una distrofia ósea, o un trastorno de forma o modelamiento del hueso provocado por una alteración del metabolismo o causa extrínseca al hueso; a diferencia de la displasia ósea, en que existen anomalías del crecimiento, es intrínseca del hueso y según Mac Kusick, (7) se deberia a un defecto enzimático. Esta osteocondrodistrofia provoca lesiones epifisiatias generalizadas, que se traducen en un enanismo

\footnotetext{
-Servicio Pediatría, Hospital Regional, Valdivia.

- "Servicio Hematología. Hospital Higueras, Talcahuano.
}

Dra. María Mena R.*, Dra. Remigia Obando."

disarmónico, el acortamiento de la columna es más acentuado que el retardo del crecimiento de los huesos Jargos. $(8,9,10)$

Los huesos largos en la diafisis, no están afectados, o ray escasamente, pero la epifisis suife una desintegración ósea que se acentúa progresivamente; así se observa genuvalgum, coxavalga. De igual manera los huesos cortos del tarso y carpo y los huesos esponjosos de lss vértebras suften igual desintegración y se cbserva plastispondilia generalizada, que ha sido enfatizada por todos los autores porque tiene una modalidad totalmente especifica $(8,9,10,11,12)$

Este síndrome es hereditario, con modalidad autosómica, recesiva, $(13,14)$ por lo que se observa con mayor frecuencia en matrimonios consanguíneos de padres sanos heterozigotos (15) Lomas, en un estudio clínico y radiográfico de 79 miembros de una familia a través de 3 generaciones, encontró 31 afectados con osteocondrodistrofia. La edad de comienzo es variable, según los autores; asi, para algunos, es después de los 3 años (16) y para otros, al empezar a caminar. (17), (18). Según Mac Kusick, (7) ya se observaría antes del año.

En una revisión practicada por Robin (19) de la literatura inglesa y americana de 29 casos, 17 habrían comenzado antes de los 18 meses, siendo 10 más notorio la xifoescaliosis. En varios casos descubrieron las anormalidades dentro de las primetas semanas, después del nacimiento. Uno de los pacientes 
de Robin mostraba alteraciones radiológicas a los 8 meses, antes de las manifestaciones clínicas. Tambiên $O$. Langer (11) concuerda con que si se observa cuidadosamente ya en las primeras semanas o meses se encontrarían sígnos clínícos y radiológicos del síndronie.

Las investigaciones efectuadas en los últinos años han reconocido en el Síndrome de Morquio, un trastorno del tejido conectivo, confirmado por Shenk (20) en biopsia de cartílago que revelaban lesiones citoquímicas de la matriz caracterizadas por lesiones amorfas fibrilares, acumulación de células espumosas con vacuolación anormal de mucopolisacáridos. También se ha demostrado que las células del tejido conectivo en cultivos sintetizan mucopolisacáridos sulfatados (21)

En cuanto a su constitución bioquímica, los mucopolisacáridos son polisacáridos que contienen, generalmente, hexosamina y están unidos con proteínas. $(22,23) \mathrm{El}$ tejido corectivo es particularmente rico en sustancias mucoides y presumiblemente la última fuente de origen de los mucopolisacáridos en vertebrados. Aún no está claramente definida la causa del defecto básico, se postulan varios defectos metabólicos, recientemente, se ha stgerido una falla en las enzimas degradativas de los mucopolisacáridos.

En cuanto a la relación entre la anormalidad del metabolismo de los mucopolisacáridos y el defecto de osificación osteocondral hay dos hipotesis, según Goidanish: (24)

$1^{0}$ Trastorno de la función de la célula por un exceso de mucopolisacáridos.

$2^{9}$ Efecto metabolico del keratostulfato en los procesos de calcificación.

Esto último parece más aceptable, ya que st: ha observado que los mucopolisacáridos pueden inhibir la nucleación de los cristales de apatita.

Normalmente se excreta por la orina escasa cantidad de mucopolisacáridos. En los viños, la cantidad aumenta con la edad. $(25,26)$. En el Sindrome de Morquio, se escretan niveles elevados de keratosulfato, $(8,9,19,21,24,27)$ o normales, según otros autores, como Matsaniotis. (28) Este sindrome corresponde a la mucopolisacaridosis IV, dentro de los clásicamente descritos; pero según Horton, (29) en 1970 existiría una 7a descrita por êl y en 1954 Ullrich, describe to que, posteriormente, ha sido llamado Sinllome de Morquio Ullrich.

\section{CASO CLINICO N: 1}

L.S.S., niña de 6 años de edad, consulta por retardo del crecimiento y deformaciones esqueléticas. Desde el año de edad, le notan las piernas desviadas hacia afuera, con separación de los pies; el esternón prominente y la columna vertebral deformada. Además, tenía opacidad de córneas. Desde los dos años presentaba dolores óseos intensos en extremidades inferiores, marcha bamboleante, cansancio al caminar, estado general conservado.

Antecedentes personales: N.P.N.T., en su domicilio, por lo que se ignora peso de nacimiento; pero consta que era un RN pequeño. En los controles médicos a los 8 meses, se refiere el diagnóstico de Raquitismo y Desnutrición, el que se repite en controles posteriores o aparece como malformaciones esqueićticas o secuelas de raquitismo. Desarrollo psícomotor normal. Recibio numerosas veces Vit. $\mathbf{D}_{2 .}$ A los 3 años, consulta en el Servicio de Ortopedia, donde se plantea el diagnóstico de: ¿Raquitismo? ¿Morquio? Se indica plartilla ortopédica y vitaminas.

Antecedentes familiares: Madre, 36 años, sana. Padre, 28 años, sano. Primos en primer grado. Embarazo: 3 abortos espontáneos, 3 hijos vivos, 1 sano, 2 enfermos.

Examen físico: Escolar con intenso retardo pondoestatural (Fig. 1). Peso: $11.500 \mathrm{~kg}$. TaJla: 80 cms. C.C.: 51 cms. C.T.: 55 cms. C.A.: $52 \mathrm{cms}$. Desarrollo psicomotor, normal, mal estado nutritivo. Desarrollo corporal, disarmónico. Normocéfalo, tronco y extremidades muy cortas. Cara alargada, corrneas opacas. Primera dentadura completa, esmalte dental delgado, amarillento, dientes separados. Cuella muy corto. Columna dorsolumbar presenta xifosis, más notoria en posición sentada. Tórax: con marcada prominencia estemal, cana- 


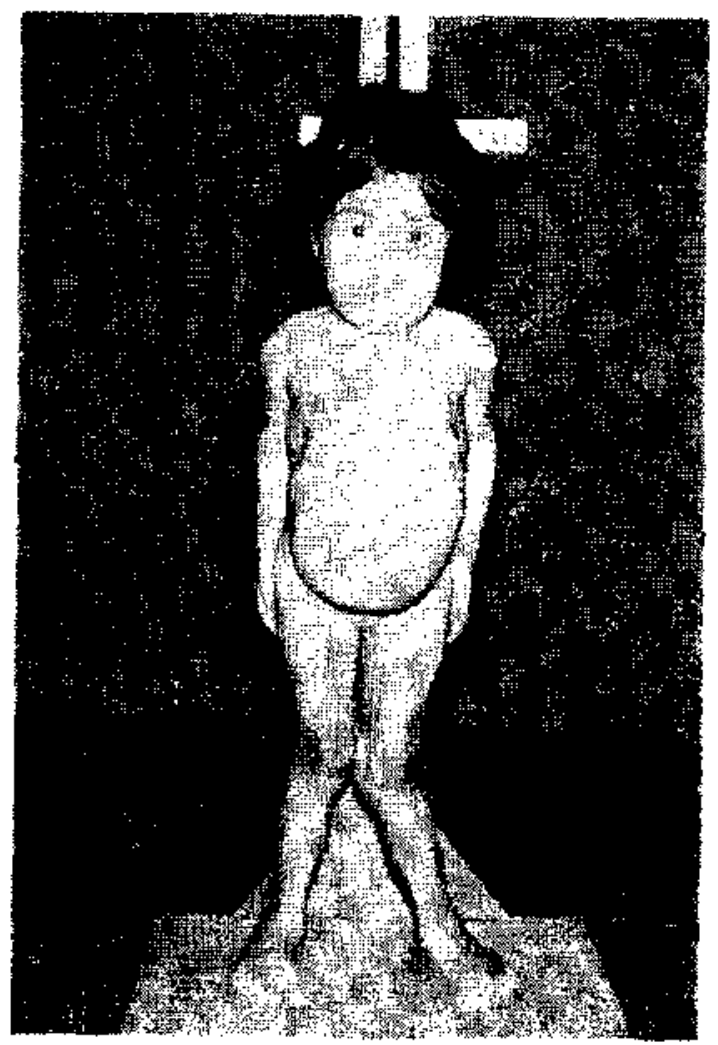

Figura 1

Caso N: 1. 6 años, gran retardo pondoestatural. Cuello y tronco, cortos. Esternon prominente. Genovalgo y pie, plano valgo bilateral.

!es de Harríson, rosario costal. Abdomen prominente, vísceras en límites normales. Extremidades cortas, en las superiores existen deformaciones óseas del húmero, cúbito $\mathrm{y}$ radio. Ensanchamiento epifisiario más marcado en muñecas, donde es muy exagerada la movilización articular. En extremidades inferiores genuvalgum intenso y pie plano valgo bilateral.

Exámenes de Laboratorio: El estudio radiológico del esqueleto revela osteocondrodistrofia generalizada. En proyección lateral de columna se observan las vértebras de escasa altura, especialmente, las torácicas que tienen además una prolongación central del hueso osificado que se extiende anteriormente, desde el cuerpo ovalado, lo que es más notorio en la región dorso lumbar, en que incluso hay deslizamiento posterior de una de las vérte- bras hipoplásicas. Espacios intervertebrale amplios. Las costillas se encuentran ensanchar das en su porcion anterior (Fig. 2). La pelv1.

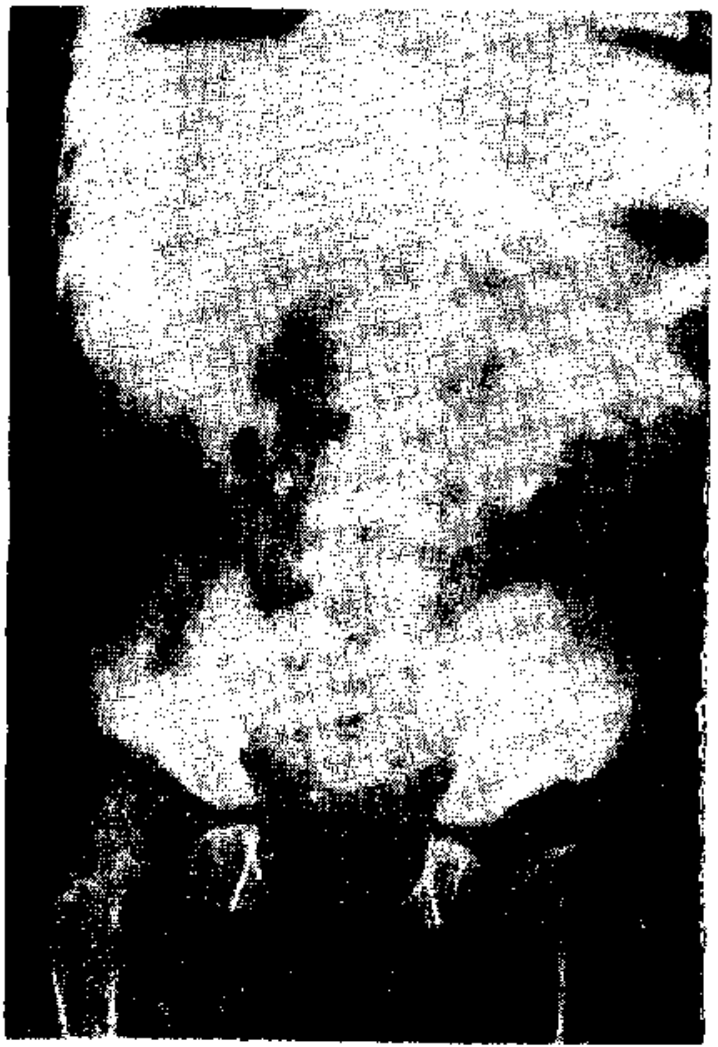

Figura 2

Radiografía de tronco. Caso № 1. Ensanchamiento anterior de costillas. Pelvis escasamente osificada. Epifisis femoral, comprimidas fragmentadas. Espacio articulat coxofemoral es amplio, cuello femoral ancho.

se estrecha a nivel de los acetábulos, está es. casamente osificada. El espacio articular coxofcmoral es amplio. La epífisis femoral aparece comprimida y fragmentada, cuello femoral ancho. La deformidad en coxavalga es acentuada. En el extremo distal del fémur, la meláfasis muestra esclerosis a lo largo de la linea del crecimiento. En la metáfasis proximal de In tibia hay oblicuidad hacia abajo y afuera de la línea articular, lo que contribuye a pro- ocar el genuvalgum. El espacio articular de la rodilla es amplio. La diăfasis del cúbito y el radio es irregular (Fig. 3). Hay acortamiento 


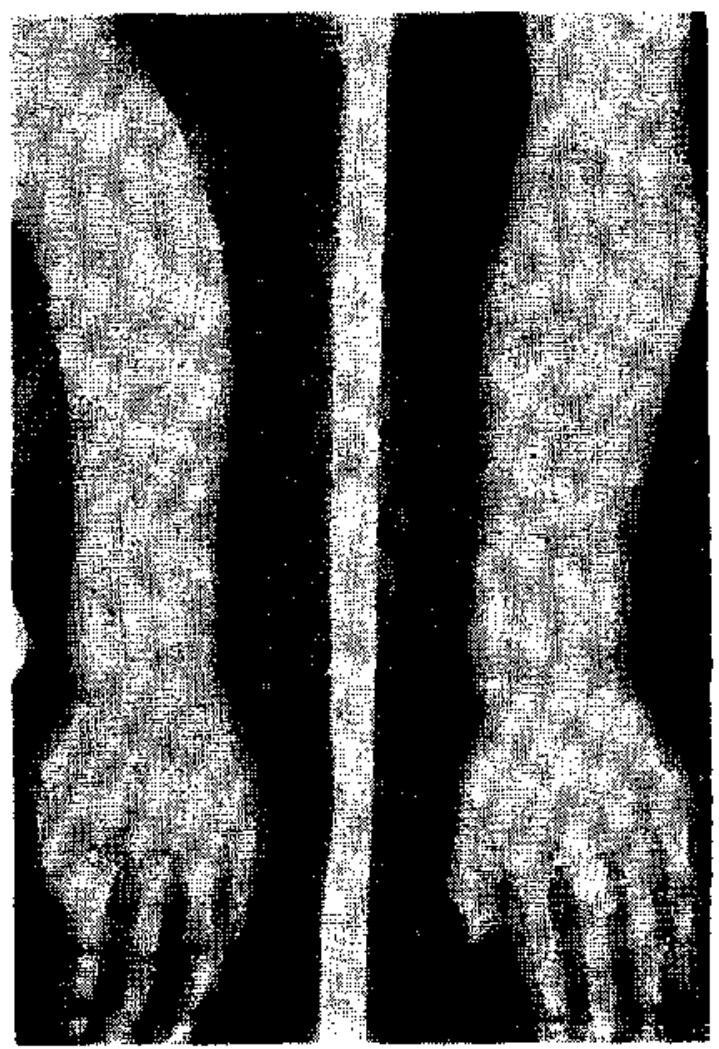

Figura 3

Extremidad superior. Caso $\mathrm{N}: 1$. Diffisis y epífisis de cúbito y radio irregulares. Cúbito acortado. Espacio articular amplio. Epiffisis proximales de metacarpíanos 2: al $5^{\circ}$ aguzados. Edad úsea retardads.

nrarcado del cúbito y las epífisis distales son irregulares. El espacio articular de la muñeca también es amplio. La osificación de los huesos del carpo está retardada. Las epífisis proximales de los metacarpianos $2^{\circ}$ al $5^{\circ}$ son de forma cónica. La silla turca de forma y tamaño normal. En el frotis sanguíneo, se observa la presencia de granulaciones en el $80 \%$ del citoplasma de los granulocitos. Se llaman granulaciones de Reilly. Lo mismo se encontró en la serie granulocítica de la médula ósea. La biopsia de piel presento metacromacia positiva, es decir, en los fibroblastos se detectó mucopolisacáridos, con tinción de azul de Toludina $\mathrm{D}$. El estudio de mucopolisacáridos urinarios eu reacción directi. cualitativa, fue positiva. Dos reacciones cuantitativas dieron cifras menores de $\mathbf{5 \%}$, aue es lo normal en 24 horas (Técnica de Hsia-Inouye). El examen oftalmológico con lámpara de hendidura, descubre opacidad correal tentre de predominio en la mitad posterior del parénquima, más denso hacia el limbo. Calcemia, fosfemia y fosfatasas alcalinas, normales. El resto de los exámenes pracicados fuleron normales.

\section{CASO CLINICO N 2}

S.S.S., lactanke de un año dos meses, se estudia a propósito de su hermana. La madre refiere que desde el primer mes de vida, le notó el pecho muy levantado y la columna vertebral con giba. Nació de parto de nalgas, de têtrmino. En etapa de RN, se observó malformaciones, como: manos y pies muy grandes; ausencia de cartílago en un pabellón auricular; sujetó la cabeza al año de edad; se sienta sin apoyo al año dos meses; habla monosílabos.

Examen físico: $10 \mathrm{kgs}$. Talla: $75 \mathrm{cms}$. CC.: $47 \mathrm{cms}$. C.T.: $48 \mathrm{cms}$. C.A.: $47 \mathrm{cms}$. Buen desarrollo pondoestatural y nutritivo. Retardo psicumotor; normocéfalo. No se aprecia opacidad corneal. Dentadura $4 / 5$, in $\mathrm{c}$ is i vos aguzados, color amarillento, con escaso esmalte. Cuello muy corto. Xifosis dorsolumbar. Esternón prominente que junta con el mentón. Abdomen bultoso, vísceras en límites normales. En extremidades existe gran flexibilidad rrticular, especialmente en muñecas.

Exámenes de Laboratorio: El estudio radiologico del esqueleto revela: osteocondrodistrofia moderada, que respeta las extremidades inferiores. Por su corta edad el aplanamiento de las vírtebras no es marcado. En proyección lateral, las vértebras tienen forma oval y en la región dorsolumbar ya se puede ver el defecto de osificación en stu parte anterior (Fig. 4). La pelvis es relativamente larga, hay osificación deficiente en la parte superior de los acetabulos, con mediana deformidad coxavalga. En el cuello femoral no se obserya la contricción media normal. La epffisis femoral está conservada. Escasa deformidad de la epífisis distal del cúbito. Osificación yormal en huesos del carpo. Las bases de Ios 


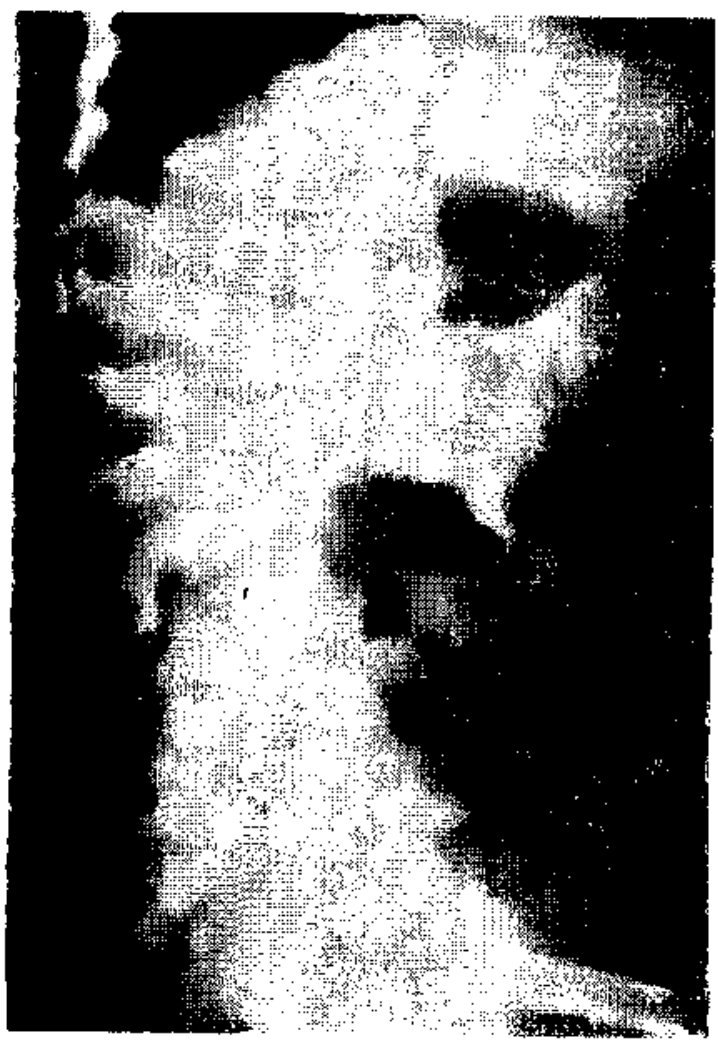

Figura 4

Vista lateral de columna vertebral. Caso $N^{0}: 2$. Lactante de 1 año 2 meses. Vértebras en forma oval. En región lumbar defecto osificación anterior de vértebras, con deslizamiento posterior de una de ellas.

metacarpianos, desde el $2^{\circ}$ al $5^{\circ}$ son conicas. La silla turca es ancha, en forma de batea. Los frotis sanguíneos muestran granulaciones de Reilly en serie granulocítica y en médula úsea. Biopsia de piel, revela metacromacia negativa. La reacción rápida cualitativa de mucopolisacáridos urinarios fue positiva, la cuantitativa, mostró $6,6 \mathrm{mg} . \%$, siendo lo normal menos de 5 mgrs.\% en 24 horas. Al examen of talmologico, tenue opacidad difusa del parénquima corneal. Resto de exámenes ce laboratorio, normales.

\section{CASO CLINICO № 3}

B.G.R, niño de 5 años 4 meses. Consulta por deformidades óseas. Al año 8 meses, por las deformaciones de su tórax, se le diagnostico: Secuelas de raquitismo. A los dos años 6 meses, un estudio radiológico reveló lesiones óseas no compatibles con raquitismo y además niveles séricos normales de $\mathrm{P}, \mathrm{Ca}$ y fosfatasos. Por insistencia en el diágnóstico anterior, recibió varias veces vitamina $D_{2} 600.000$ unidades. No presentó dolores 6seos; pero tenía marcha banboleante.

Antecedenies personales: N.P.N.T., en Hospital Regional de Valdivia. Peso de nacimiento $3.200 \mathrm{~kg}$. Se controló en forma regular; sujetó la cabeza entre 5 y 4 meses; se sento sin apoyo a los 8 meses; carninó al año 6 meses; hablo a los 12 meses.

Antecedentes familiares: Padres sanos sin parentesco faniliar. Dos embarazos. Ningún aborto. Una hermana sana, de 3 años de edad.

Examen físico: $15 \mathrm{kgs}$. de peso. Talla: 90 crns. C.C.: 54 cms. C.T.: 53 cms. C.A.: 51 crns. Gran retardo pondoestatural. Cabeza grande, en proporción al cuerpo. Cara alargada. Cuello corto. Tórax con esternón prominente que se toca en el mentón. Tronco corto, columna vertebral sin xifosis, pero no existe la curvatura lumbar normal. Abdomen, vísceras en límites normales. Extremidades superiores, ensanchamiento epifisiario en muñecas y codos, hiperlaxitud articular. Extremidades inferiores, genovalgo, pie plano valgo. Estudio radiológico del esqueleto: manos con falanges cortas y anchas. Cúbito corto y epífisis irregulares; radio, con epífisis irregulares, gian espacio interarticular. Edad osea retardada. Columna, platispondilia generalizada; vértebras en forma de "cabeza de pescado". Pelvis, estrechada a nivel de acetábulos; cabeza femoral aplastada. Línea de Shenton discontinua. Examen oftalmológico con lámpara de hendidura, confirmó la opacidad de córnea. Mucopolisacáridos urinarios, $29,26 \mathrm{mg}$. en 24 horas (normal adultos: $20 \mathrm{mg}$.). Relación Carbazol Orcinol: 2,53, descarta la presencia de Keratan dermatan sulfato. Al hemograma existian granulaciones de Reilly en serie granulocítica y médula ósea. A la biopsia de piel, metacromacia (-). El examen psicológico reveló debilidad mental definida. 


\section{COMENTARIO}

Se presentan 3 casos clínicos, dos son hermanos, hijos de matrimonio consanguíneo, que tienen cono rasgo clínico principal: malformaciones esqueléticas con una radiologia específica. Además, aparecen rasgos clínicos y de laboratorio que reflejan acúmulo de mucopolisacáridos como: opacidad corneal, granulaciones de Reilly en sangte y médula, eliminación discretamente aumentada de mucopolisacáridos en un niño y normal en otro. Metacromacia positiva en piel. Basados en estos signos, se plantea el diagnóstico de Síndrome de Morquio.

En el tercer caso clínico no había consanguinidad paterna, pero en general, coincidieron los ragos clínicos y de laboratorio y se demostró una elevađa excreción de mucopolisacáridos urinarios.

El diagnóstico diferencial se debe hacer, en primet lugar, con raquitismo. $(16,17,30$, $31,32)$ En la literatura nacional y extranjera la mayoría de los casos de Morquio han sido referidos en un primer momento como raquitismo. Aparentemente, el diagnóstico diferencial es fácil, dado que, en el raquitismo y especificamente, en el tardio o vitaminorresistente, se observan lesiones radiológicas específicas, con alteración de los niveles séricos de $\mathrm{Ca}, \mathrm{P}$ y $\mathrm{FA}$, además de otras alteraciones humorales propias de la etiología del raquitismo. En el Morquio, todos estos índices son normales y presentan aspectos clínicos y radiológicos totalmente propios. Sin embargo, y a pesar de la escasa frecuencia de este síndrome, no se debe olvidar, cuando nos enfrentamos a un posible raquitismo tardio.

También cabe hacer diagnóstico diferencial con otros mucopolisacáridos; el más parecido es el Síndrome de Hurler, $(16,33,34)$ en el que existe facies grotesca o de gárgola, gran retardo mental, signos de compromiso visceral, opacidad corneal, sordera, compromiso valvular cardíaco, hepatoesplenomegalia, tigidez articular, xifosis lumbar progresiva, acortamiento notable de tronco y extremidades. A menudo coxavalga, genovalgum, pie plano, es decir, que en cuanto a las malformaciones es- queléticas, existe semejanza entre los dos cuadros, pero radiológicamente, existen diferencias claras $(10,14,35)$ y así, en el Hurler hay ensanchamiento de las diáfisis de los huesos largos con trabeculación defectuosa. La platispondilia no es generalizada. Los metacarpianos son cortos y anchos, las falanges tienen forma de bala, silla turca ensanchada como batea. Sin embargo, a pesar de coincidir los rasgos clínicos y radiológicos presentados por estos hermanos, con la enfermedad descrita por Morquio y Brailsford, llama la atención que presentan signos descritos, específicamente, en enfermedad de Hurler, tales como granulaciones de Reilly, que bioquímicamente se han calificado como acúmulo de mucopolisacáridos y fueron descritos en 1941 por Reilly (36) en letcocitos de pacientes con Hurler.

En la literatura, estas granulaciones de Reilly, han sido descritas en numerosos pacientes con Síndrome de Morquio. $(7,8,19$, 37, 38) En 1954 Wiedemann sugirió el nombre de Morquio-Ullrich al Síndrome de Morquio, con uno o más de los aspectos extraesqueléticos de Hurler. Varios autores han clasificado a sus pacientes en esta nueva enfermedad: Goidanish, Zellwegger, Rask, Dyggve, Torelli. (24, 37, 39, 40, 42) Sin embargo, Maroteaux y Lamy, Langer, Robin y otros, plantean, que una división entre Morquio Brailsford y Morquio Ullitich, es artificial y que una cuidadosa investigación clínica de los pacientes, puede invariablemente, revelar tales cambios o signos de mucopolisacaridosis generalizada y si se hiciera un examen oftalmológico con lámpara de hendidura, se descubriría, constantemente, opacidad corneal que aumenta progresivamente con la edad.

En estos pacientes, se ha demostrado que eliminan keratosulfato urinario, según Robin (19) y otros autores; otros pacientes han eliminado condroitinsulfato $B$ y ácido hialurónico, según Clausen Dyggve. (41) Matsaniotis, mostró en un Síndrome de Morquio con granulaciones de Reilly, excreción normal de mucopolisacáridos. Torelli (42, efectú cariograma en pacientes clasificados como MorquioUllrich y observo que existia en todos los cromosomas del grupo D, satélites en los brazos 
cortos. Llama la atención la metacromacia positiva de piel en la biopsin del mayor de los niños, Shanon Danes (43) en investigaciones sobre metacromacia de piel, como signo genetico para estudiar mucopolisacaridosis, describió metacromacia $(+)$, en sujetos afectados con Sindrome de Hurler y Hunter y sus familiares heterozigotos; solamente hubo metacromacia en aquellos Morquios que presentaban ntros signos de acúmulo de mucopolisacáridos extraesquelético: granulaciones de Reilly, ensanchamiento de la silla turca, retardado mental. Este autor, incluso, concluye que los Morquios sin cambios somáticos que comprometan otros tejidos, además del esqueleto, no pueden ser clasificados como mucopolisacaridosis generalizada. (44) Todas estas variaciones signologicas nos llevan a pensar que esto plantea, como dice Mac Kusick, que se trata de similitudes fenotipicas con genotipos distintos.

En revisiones de literatura, existen numerosos casos de Morquio Brailsford; ya en 1941 Whiteside refiere 71 casos publicados.

\section{RESUMEN}

Se presentan tres casos clínicos de Síndrome de Morquio. En los dos hermanos, se trataba ce herencia autosómica recesiva.

Clínicamente se observan malformaciones óseas y se demuestra radiologicamente una osteocondrodistrofia generalizada.

También el examen clínico y de laboratorio muestra signos de acúmulo de mucopolisacaridos, opacidad corneal, grantulaciones de Reilly, metacromacia de piel.

Se revisa la literatura y se resuelve no clasificar este síndrome como Morquio-Ullrich, sino como Morquio Brailsford, con una investigación clínica y de laboratorio exhaustiva.

\section{SUMMARY}

Three clinical cases of Morquio's Syndtome are presented. Two of them were brothers with autosomic recesive inheritance.

Bone malformations were clinically found out together with radiologycal generalized ssteochondrodystrophies.
Other clinical and laboratory findings were: signs of anounts of mucopolysaccharjdes, corneal opacity, Reilly's granulations and netachromatia of the skin.

After reviewing the disposable literature, the author resolved to not clasify these cases like Morquio Ullrich Syndrome, but like Morquio Brailsford one, with an exhaustive clinical and laboratory investigation.

\section{REFERENCIAS}

1. Morquio, L.: Arch. de Med. d. enfants. 32; 129, 1929.

2. Morqquio, L.: Arch. de Med. de enf. 38: 5, 1935.

3. Brailsford. J.: The Am. J. of Sur. 7: 400-410. 1929.

4. Freedman, J.: Am. I. Dis. Child. 55: 343-355, 1938.

5. Langer, L.O.: Growth. Clin. Ped. 3: 142, 1969.

6. Dale, T.: Acta Radiol. 12: 337, 1931.

7. Mc Kusick, V.A.: Am. J. of M. 47: 730, 1969.

8. Maroteatux, P. et Lamy, M.: La Presse Médicale. 71: 2091, 1963.

9. Rusker, A., Evans, L. anđ Langer, L.: J. Ped. Diciembre 1970, pág. 1039.

10. Soto, /.: Archivos de Pediatría del Uruguay. Julio de 1965, pág. 448.

11. Langer, L. and Carey, L.: The Am. J. of Roentgenology. 97: 1, 1966.

12. Feldenon, N. y Davenport, M.E.: Arch. Dis. Child. 26: 279, 1951.

13. Sitith, D.: Atlas de Malformaciones Somáticas en el Niño, 1972.

14. Maraleaux, P. and Lamy, M.: The J. of Ped. 67: 312, 1965.

15. Lomas, J, and Boyle, A.C.: Lancet, 2: 430, 1959.

16. Bustamante, W., Doberti, A.: Usteocondrodistrofie deformante. Enfermedad de Morquio.

17. Einhorn y al.; Am. I. of Dis. of Children. 536, 1946.

18 Ruggles, H.E.: Am. J. of Roentgenology. 25: 90, 1931.

19. Robins, M., Stevens, H., Linker, A.: Morquio disease. J. of Ped. 62: 881, 1963.

20. Schenk, E. and Haggerty, J.: Pediatrics. 34: 839, 1964.

21. Danes, Sh., Grossman, H.: The Am. I. of Med. Noviembre 1969, pág. 708.

22. Yoy Ballet, A.: Connective tissue polysaccharide metabolism and the pathogenesis Arch. in Internal. Med. Vol. 13: 33, 1967.

23. Dorfman, A.; Pediatrics. 22: 576, 1958.

24. Goidanich, I. and Lenzi, L.; The J. Bone and Joint Surgery. 46: 734, 1964. 
25. Teller, W., Burke, E., Rosevear, l.: Urynary excretion of acid mucopolysaccharides in normal Children and patients with gargoylism.

26. Rich, C., Ferrant, N.: J. Lab. Clin. Med, 50: 686, 1967 .

27. Sanfitippo, S. and Good, R.A.: J. of Pediat. 61: 296, 1962.

28. Matsaniotis, N., Kattanies, C., Lehmann, H.: Arc. Dis, Child, 42: 652, 1967.

29. Horton, W. and Schimke, N.: J. Pediat, 77: 252, 1970.

30. Russo, P.: Radiology. 41: 42, 1943.

31. Einhorn, N., Moore, I.: Am. I. Dis. Children. 61: $776,1941$.

32. Meyer, H., Brenneman, I.: Am. J. Dis. of Children. 43: 123, 1942,

33. Horrigan, D. and Bocker, D.: The Am. J. of Roentgenology. 68: 473, 1961.

34. Palacios, E. y Brunser, O.: Pediatria. 5: 318 , 1962.
35. Fairfank, T.: The $\mathbf{~}$. of bone and Joint surgery. 31: 291, 1949.

36. Reilly. W.: Am. J. Dis. of Children. Septiembre 1941 , pág. 489.

37. Zellweger, H., Ponsetx, J., Pedrini, V.: The J. of Ped. 59; 549, 1961.

38. Link, A. y Gassorla, E.: Rev. Chil. Ped. 1966, pág. 779.

39. Rask, M.; The J. of Bone and Joint surgery. 45 A: 561, 1963.

40. Dyggve, H., Melchior, J. and Clausen, I.: Arch. of Dis. of Children. 37: 525, 1963.

41. Clauser, 1., Dyggue, H.: Arch. Dis. Clildren. 38: 364, 1963.

42. Torcli, M.: La Pediatria. II: 264, 1969.

45. Danes, S. and Searn. A.: Lancet. 1: 241, 1967.

44. Batzá, I., Contreras, I.: Las mucopolisacarido. sis. Enfermedades genéticas y citogenéticas en el nin̄o. Andrés Bello, 1974. 